\title{
Autori / Contributors / Auteurs
}

Damiano Acciarino è nato a Roma nel 1986. Ha completato la sua formazione tra l'Università Cattolica del Sacro Cuore di Milano e l'Università Ca' Foscari di Venezia, dove adesso detiene una Marie Curie Fellowship congiuntamente con la University of Toronto. Si occupa di antiquaria rinascimentale e tradizione classica nel Cinquecento.

Barbara Alfano teaches Italian at Bennington College. She holds a Ph.D. in Comparative Literature and specializes in contemporary Italian narrative, with a focus on representations of America, issues of identity and the individual, love, and women's writing. She is the author of The Mirage of America in Contemporary Italian Literature and Film (University of Toronto Press, 2013). She has published essays in several journals, among which Italica, Forum Italicum, and Italian Quarterly. Her three latest essays discuss the work of Sibilla Aleramo, Elena Ferrante, and Oriana Fallaci. She is the co-editor of Italian Quarterly's special issue "Projecting Americanism Abroad: Italy in the Cold War" (2019), for which she also wrote the essay "Oriana Fallaci: Tales of America on the Road to Women's Emancipation." She curates a colloquium on Elena Ferrante hosted by Stanford University's on-line literary salon, Arcade.

Eugenio Bolongaro is Associate Professor of Italian Studies in and Chair of the Department of Languages, Literatures, and Cultures at McGill University in Montreal. A native of Italy, he was trained at the University of British Columbia (BA in English; LLB) and then at McGill University (PhD in Comparative Literature) where he has taught since 2003. His book Italo Calvino and the Compass of Literature was published by the University of Toronto Press in 2003. In 2009, he co-edited the volume Creative Interventions: The Role of Intellectuals in Contemporary Italy. He has published on the theory of genres, the fantastic and the interface between ethical philosophy and literature. His literary contributions include several articles on Italo Calvino, post-WW II Italian cinema (Bertolucci, Visconti), contemporary Italian fiction (Tondelli, Nove) and the contemporary US author Thomas Pynchon. His most recent work focuses on the exploration of a post-humanist perspective in contemporary European narratives both cinematic and literary. 
Eveljn Ferraro is Assistant Professor of Italian Studies at Santa Clara University. She holds a Ph.D. in Italian Studies from Brown University. Her teaching and research interests include Italian Diaspora Studies (with a focus on North America), postcolonial Italy, liminal spaces in literature and cinema, and the relationships between national belonging and transnational identities resulting from old and new migrations from and to Italy and its global connections. She has published essays on the American experience of Italian Jewish writer Ebe Cagli Seidenberg and is currently investigating refugee women, coalition politics, and affect in Cagli Seidenberg's testimonial writing. She is also working on a project titled "Giuseppe Pitrè: Transcribing Sicily for the Italian Nation." With Laura Dolp she co-authored a book chapter and an article on Gabriella Ghermandi's music and storytelling. She is a Book Review Editor for Altreitalie, an International Journal of Studies on Italian Migrations in the World.

Gianmarco Gallotta, Ph.D, è attualmente responsabile delle relazioni internazionali all'IAE - Université Savoie Mont Blanc (Francia). I suoi interessi di ricerca si focalizzano sul romanzo italiano del secondo Novecento e sul rapporto tra letteratura e cinema. La sua tesi in cotutela tra l'Université de Lorraine e l'Università di Salerno, discussa nell'autunno 2014, è stata pubblicata da Chemins de tr@verse con il titolo Antonio Tabucchi:un baule di personaggi. Attualmente sta lavorando a diversi progetti didattici e di ricerca legati allo sviluppo delle relazioni tra università europee ed extraeuropee.

Gianpasquale Greco è nato a Napoli nel 1987. Dottore di ricerca all'Università degli Studi di Napoli "Federico II" e professore di ruolo di Storia dell'Arte negli istituti superiori, ha pubblicato diversi contributi scientifici sul Barocco, particolarmente sulle sepolture d'artista, ed ha approfondito la filologia delle fonti storico-artistiche del XVII e XVIII secolo. Giornalista presso l'Espresso napoletano, ha pubblicato per Rogiosi Tales from New York. Lettere di un artista napoletano, 1909-1910 (2016), Notizie del bello dell'antico e del curioso della città di Napoli. Edizione critica della ristampa del 1792, con le aggiunte del 1724 e del 1758-59 (2018), e per lo stesso editore coordina la collana editoriale "Il merito di Napoli".

Alessandro Martini, agrégé d'italiano e dottore in letteratura italiana contemporanea, è professore associato presso l'Université Jean Moulin Lyon 3. È autore di una monografia su Beppe Fenoglio (Beppe Fenoglio, témoin et romancier 
de la Résistance, 2012). Si occupa di letteratura e memorialistica resistenziale, a cui ha dedicato diversi saggi (Fenoglio, Revelli), e della scrittura della storia nelle finzioni letterarie. Ha studiato questo tema nell'opera di Salvatore Quasimodo, Andrea Camilleri e Giorgio Bassani. Ha co-diretto un volume sulla ricezione delle opere di quest'ultimo autore: Da Ferrara a Buenos Aires. Contributi sulla ricezione italiana e internazionale di Giorgio Bassani (2018). Si occupa attualmente di storia dell'editoria contemporanea, in particolare delle case editrici legate al movimento partigiano.

Michele Ronchi Stefanati è "Professor Eduardo Saccone PhD Scholar" in Italian Literature a University College Cork (UCC). Si è laureato in Filologia medievale e umanistica all'Univerisità di Bologna e ha ottenuto la laurea magistrale in Italianistica presso la medesima università con una tesi in Filologia italiana dal titolo "Riscritture. Studio sul Furioso del '32 alla luce delle due precedenti edizioni e degli autografi ariosteschi”. Dopo un periodo come affiliate student a University College London (UCL), si è dedicato principalmente al rapporto tra la letteratura italiana contemporanea e l'impegno, con particolare riferimento all'opera di Gianni Celati, su cui ha pubblicato diversi saggi. È stato l'organizzatore del convegno "Gianni Celati. Tradition, Translation, Rewriting", tenutosi a Dublino e a Cork nel 2016, a cui ha preso parte lo stesso Celati.

Camilla Skalle (PhD) è professore associato di letteratura italiana nel Dipartimento di lingue straniere all'Università di Bergen, dove insegna cultura e letteratura italiana, didattica di lingue straniere e teoria letteraria. I suoi interessi di ricerca includono la letteratura mitologica, la narrativa migrante e postcoloniale, la letteratura e le teorie ecocritiche, le filosofie femministe. Le sue pubblicazioni più recenti sono apparse sulle riviste borderlands, Neophilologus, BeLLs e Acta Didactica. 